\title{
Análise do perfil do estilo de vida de professores universitários e sua relação com a obesidade como fator de risco para morbidade
}

Analysis of the lifestyle profile of university professors and its relationship to obesity as a risk factor for morbidity

Análisis del perfil de estilo de vida de los profesores universitarios y su relación con la obesidad como factor de riesgo de morbilidad

\section{Resumo}

A obesidade representa um preocupante problema de saúde pública por estar aumentando de maneira exorbitante, o que, consequentemente, eleva o risco para as morbidades associadas a ela, além de ser a principal causa de óbitos em pessoas com menos de 60 anos devido à COVID-19. O objetivo do presente estudo foi avaliar, por meio de questionário na plataforma do Google Forms, a qualidade de vida dos professores dos cursos de graduação do Centro Universitário de Patos de Minas (UNIPAM), verificando a importância dos exercícios físicos, tanto resistidos quanto aeróbios, no combate à obesidade como fator de risco para morbidade, bem como compará-los. Trata-se de um estudo transversal descritivo qualitativo-quantitativo, realizado através do Microsoft Excel 2016 e os resultados foram apresentados por meio de gráficos e tabelas. A amostra foi composta por 80 professores universitários do UNIPAM, em que $63,7 \%$ eram do sexo feminino e $36,3 \%$ eram do sexo masculino. Foi verificado que a maioria dos docentes, tanto dos cursos da área da saúde quanto dos cursos de outras áreas, se apresentaram eutróficos, porém os da área da saúde, relacionando-os com o IMC, possuem maior sobrepeso e obesidade, mas os desta área tiveram amostra maior (n 54). Diante dos dados levantados na pesquisa, conclui-se que ambos os cursos possuem a maioria da população eutrófica, mas ainda há uma quantidade significativa com sobrepeso e obesidade, além de que somente 42,5\% não possuírem nenhuma patologia, sendo proposto que mais estudos sejam realizados para maiores resultados e conhecimentos.

Palavras-chave: Obesidade; Morbidades; Exercício físico; COVID-19.

\begin{abstract}
Obesity represents a worrying public health problem as it is increasing exorbitantly, which, consequently, increases the risk of morbidities associated with it, in addition to being the main cause of death in people under 60 years old due to COVID-19. The aim of this study was to evaluate, through a questionnaire on the Google Forms platform, the quality of life of professors in the undergraduate courses at the University Center of Patos de Minas (UNIPAM), verifying the importance of physical exercise, both resistance and aerobic, in combating obesity as a risk factor for morbidity, as well as comparing them. This is a descriptive qualitative-quantitative cross-sectional study, carried out using Microsoft Excel 2016 and the results were presented using graphs and tables. The sample consisted of 80 university professors from UNIPAM, in which $63.7 \%$ were female and $36.3 \%$ were male. It was found that most professors, both from courses in the health area and from courses in other areas, were eutrophic, but those in the health area, relating them to BMI, are more overweight and obese, but those in this area had a larger sample (n 54). Given the data collected in the research, it is concluded that both courses have the majority of the eutrophic population, but there is still a significant amount of overweight and obesity, and only $42.5 \%$ do not have any pathology, and it is proposed that further studies are carried out for greater results and knowledge.
\end{abstract}

Keywords: Obesity; Morbidities; Physical exercise; COVID-19.

\section{Resumen}

La obesidad representa un problema de salud pública preocupante ya que está aumentando de manera exorbitante, lo que, en consecuencia, aumenta el riesgo de morbilidades asociadas a ella, además de ser la principal causa de muerte en personas menores de 60 años por COVID-19. El objetivo de este estudio fue evaluar, a través de un cuestionario en 
la plataforma Google Forms, la calidad de vida de los docentes de los cursos de pregrado del Centro Universitario de Patos de Minas (UNIPAM), verificando la importancia del ejercicio físico, tanto de resistencia como aeróbicos, en la lucha contra la obesidad como factor de riesgo de morbilidad, así como en su comparación. Se trata de un estudio descriptivo, cualitativo-cuantitativo, transversal, realizado con Microsoft Excel 2016 y los resultados se presentaron mediante gráficos y tablas. La muestra estuvo formada por 80 profesores universitarios de UNIPAM, de los cuales el $63,7 \%$ eran mujeres y el 36,3\% eran hombres. Se encontró que la mayoría de los profesores, tanto de cursos del área de la salud como de cursos de otras áreas, eran eutróficos, pero los del área de salud, relacionándolos con el IMC, tienen más sobrepeso y obesidad, pero los de esta área tienen una mayor muestra (n 54). Dados los datos recogidos en la investigación, se concluye que ambos cursos tienen la mayoría de la población eutrófica, pero aún existe una cantidad significativa de sobrepeso y obesidad, y solo el $42,5 \%$ no tiene ninguna patología, y se propone que más se realizan estudios para obtener mayores resultados y conocimiento.

Palabras clave: Obesidad; Morbilidades; Ejercicio físico; COVID-19.

\section{Introdução}

A obesidade é uma doença caracterizada por um complexo distúrbio metabólico e está associada a diversas complicações, como doenças cardiovasculares, diabetes, disfunção renal, disfunção hepática e câncer, o que resulta em diminuição da qualidade de vida (Manna, \& Jain, 2015). No Brasil, o número de pacientes com obesidade aumentou muito nos últimos anos, sendo estimado que mais de $50 \%$ da população esteja na faixa de sobrepeso ou de obesidade. A Organização Mundial de Saúde estima que, em 2025, cerca de 700 milhões de adultos no mundo estejam com obesidade e 2,3 milhões com sobrepeso (ABESO, 2021).

A interação de fatores genéticos, emocionais, ambientais e de estilo de vida refletem diretamente na obesidade, o que afeta vários sistemas e órgãos, além de aumentar o risco de desenvolvimento de doenças cardiovasculares, gástricas, geniturinárias, musculoesqueléticas, entre outras (ABESO, 2016; Garvey et al., 2016; Jensen et al., 2013). Essas complicações podem causar outros problemas, como a piora da qualidade de vida, altos encargos psicossociais e econômicos, além do aumento de morbidades (Garvey, et al., 2016; Jensen, et al., 2013).

De acordo com a Pesquisa Nacional de Saúde de 2019 e a Pesquisa de Orçamentos Familiares (POF) de 2002-2003, cerca de 7,4 milhões de pessoas, o que equivale a 33,7\%, de 18 a 24 anos estavam com excesso de peso em 2019 e $10,7 \%$ com obesidade. Já quando se observa os indivíduos com faixa etária entre 40 e 59 anos, essa prevalência mais que dobra, subindo para 70,3\% para os com excesso de peso e 34,4\% para os com obesidade, o que representa 39,5 milhões de pessoas. Em adultos de 18 anos ou mais, o déficit de peso é representado pelo Índice de Massa Corporal (IMC) abaixo de 18,5 Kg/m², o sobrepeso pelo IMC igual ou acima de $25 \mathrm{~kg} / \mathrm{m}^{2}$ e a obesidade igual ou superior a $30 \mathrm{~kg} / \mathrm{m}^{2}$, considerando que a obesidade é um subgrupo do sobrepeso (OMS, 2020).

Os benefícios da perda de peso corporal são sistêmicos, como diminuição do risco cardiovascular, prevenção e controle da diabetes tipo 2, atenuação da resistência insulínica, redução dos níveis de pressão arterial, melhora dos níveis lipídico, queda da mortalidade por doenças cardiovasculares, além da redução do tecido adiposo e da circunferência abdominal, o que leva à diminuição de marcadores inflamatórios sistêmicos e aumento da aptidão física (Garvey, et al., 2016; Hansen, et al., 2018 ; NHMRC, 2013). Os exercícios físicos têm sido considerados pelos órgãos internacionais como um componente fundamental na intervenção do controle do peso corporal em virtude do gasto calórico por ele provocado, seja durante o exercício ou após o mesmo (Cavalcanti, et al., 2010).

Fernandez, et al. (2004) observaram que tanto o exercício aeróbico quanto o anaeróbico foram efetivos no tratamento da obesidade. O treinamento aeróbico é considerado como um tipo de exercício eficaz para aumentar o gasto energético e o consumo de oxigênio pós-exercício (Excess Post-exercise Oxygen Consumption - EPOC), entretanto, estudos têm demonstrado que a prática de exercícios resistidos também pode exercer uma influência positiva sobre estas variáveis. Evidências sugerem que os exercícios resistidos podem estimular um EPOC significativo para o controle do peso corporal e, alguns estudos têm reportado um EPOC significativamente maior em resposta aos exercícios resistidos quando comparado ao exercício aeróbico 
(Poehlman, et al., 2000). O treinamento com exercícios resistidos promove o gasto calórico e aumenta as concentrações sanguíneas de HDL, conhecido como colesterol bom (Hansen, et al., 2018). Além disso, ao agregar exercícios resistidos com programas de restrição calórica, a diminuição de massa gorda e a preservação da massa livre de gordura são mais eficientes (Batsis, et al., 2017).

Portanto, tendo em vista que a prevalência de excesso de peso e de obesidade está aumentando em um ritmo alarmante em muitos países, inclusive no Brasil, o presente estudo pretendeu elucidar a análise do perfil do estilo de vida dos professores universitários do Centro Universitário de Patos de Minas (UNIPAM) e sua relação com a obesidade como fator de risco para morbidade, já que é esperado que esses indivíduos, principalmente da faixa etária estudada, mesmo sendo muito ocupados devido ao trabalho, praticam exercícios físicos devido aos seus inúmeros benefícios. Entretanto, ainda podem ter alguns que possuem distúrbios, como a obesidade, que se não identificados e solucionados pelos profissionais da saúde, vão comprometer o tratamento e a melhora dessa patologia (Zabatiero, et al., 2015).

\section{Metodologia}

Trata-se de uma pesquisa de campo, de abordagem transversal descritiva qualitativa-quantitativa, cuja classificação se dá por finalidade básica pura, por objetivo descritiva e modalidade bibliográfica (Estrela, 2018).

O presente estudo apresenta uma pesquisa de campo, que foi estruturado da seguinte maneira: escolha do tema, levantamento do assunto da pesquisa, definições de parâmetros de busca de inclusão e exclusão dos artigos, elucidação das referências citadas, análise dos estudos, avaliação dos principais resultados e a formulação do artigo complementando as etapas.

A pesquisa foi guiada pela questão norteadora: Professores universitários do Centro Universitário de Patos de Minas (UNIPAM), mesmo sendo muito ocupados devido ao trabalho, praticam exercícios físicos e isso afeta diretamente sua qualidade de vida, além de auxiliar na prevenção e no combate da obesidade e nos fatores de risco para morbidade?

A busca dos estudos ocorreu no período de fevereiro a outubro de 2021. Os critérios de inclusão foram professores do Centro Universitário de Patos de Minas (UNIPAM) de 40 a 60 anos, do sexo feminino e masculino, que aceitaram participar da pesquisa de acordo com o TCLE, sendo contactados via e-mail e via WhatsApp. Foram analisados o IMC, através do cálculo do peso dividido pela altura ao quadrado, a Escala de Silhueta de Stunkard, se praticavam ou não exercício físico, as patologias que possuíam, se já tiveram COVID-19 e se seu estilo de vida afetou diretamente em sua recuperação, além de questões de opinião variadas. Foram excluídos professores que estiveram com atestado médico no período da coleta de dados e que os impediu de realizar o projeto, como por exemplo por questões psicológicas, ou caso tenham desistido de responder o questionário.

A Escala de Silhueta de Stunkard é uma escala composta por 9 silhuetas esquemáticas propostas por Stunkard, Sorensen e Schulsinger (1983) que avalia distorções na imagem corporal. Nessa escala, as silhuetas aumentam gradualmente suas dimensões com intervalo de IMC de 2,5 kg/m2 em cada imagem. Cada voluntário marcará a imagem corporal que estiver de acordo com sua estrutura física. 
Figura 1 - Padrões de Silhuetas.
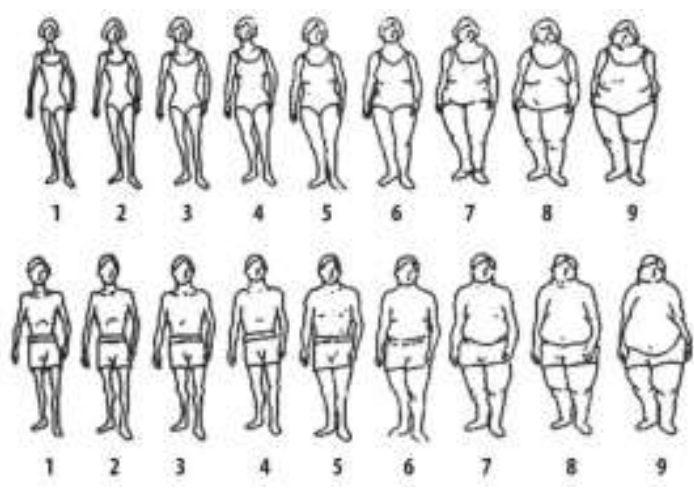

Fonte: Stunkard, Sorensen e Schulsinger (1983).

O estudo foi submetido ao Comitê de Ética em Pesquisa com Seres Humanos do Centro Universitário de Patos de Minas - UNIPAM, e após ter sido aprovado, foi encaminhado o questionário para os professores universitários que, caso aceitassem o Termo de Consentimento Livre e Esclarecido, poderiam respondê-lo. Foi realizada uma coleta de dados através desse questionário autoaplicável.

Os dados foram analisados por meio de estatística descritiva. Os resultados foram apresentados por meio de gráficos e tabelas (Microsoft Excel 2016), separadas por sexo, feminino e masculino, para analisar e verificar a importância dos exercícios físicos, tanto resistidos quanto aeróbios, no combate à obesidade como fator de risco para morbidade, e, posteriormente, foram comparados.

Devido à grande lacuna no que diz respeito aos estudos voltados a obesidade e as morbidades associadas a ela, percebeuse que eram necessárias mais pesquisas acerca do tema, principalmente na faixa etária abordada.

\section{Resultados e Discussão}

A amostra foi composta por 80 professores universitários do UNIPAM, sendo 67,5\% (n 54) de cursos da área da saúde e $32,5 \%$ (n 26) de cursos de outras áreas, na qual 63,7\% (n 51) eram do sexo feminino e 36,3\% (n 29) do sexo masculino.

A média de peso foi de $74,35 \mathrm{Kg}$, de altura $1,68 \mathrm{~m}$ e de IMC $26,10 \mathrm{Kg} / \mathrm{m}^{2}$ (Tabela 1), sendo que foram distribuídos em idades de $<40$ anos, $40-49$ anos e $\geq 50$ anos (Tabela 2), de ambos os sexos.

Tabela 1 - Caracterização das variáveis dos participantes.

\begin{tabular}{|cccc|}
\hline & Média & Desvio Padrão & Mediana \\
\hline Peso & 74,35 & 17,19 & 73,5 \\
Altura & 1,68 & 0,09 & 1,68 \\
IMC & 26,10 & 4,91 & 25,59
\end{tabular}

Fonte: Autores (2021).

Tabela 2 - Idade dos participantes.

\begin{tabular}{|cc|}
\hline \multicolumn{2}{|c|}{ Idade } \\
\hline$<40$ anos & 30 \\
$40-49$ anos & 24 \\
$\geq 50$ anos & 26
\end{tabular}

Fonte: Autores (2021). 
A avaliação do Índice de Massa Corporal (IMC), foi classificada, segundo a Organização Mundial da Saúde (OMS2020) em quatro categorias: déficit de peso (abaixo de $18,5 \mathrm{Kg} / \mathrm{m}^{2}$ ), normal (entre $18,5 \mathrm{Kg} / \mathrm{m}^{2}$ e $25 \mathrm{Kg} / \mathrm{m}^{2}$ ), sobrepeso (igual ou acima de $25 \mathrm{~kg} / \mathrm{m}^{2}$ ) e obesidade (igual ou superior a $30 \mathrm{~kg} / \mathrm{m}^{2}$ ), considerando que a obesidade é um subgrupo do sobrepeso.

Os valores dos percentuais resultantes do IMC relacionado com os profissionais do UNIPAM que lecionam nos cursos da área da saúde e os que lecionam nos cursos de outras áreas apontaram que 31,25\% dos docentes dos cursos da área da saúde possuem IMC considerado normal e, somente $11,25 \%$ dos docentes dos cursos de outras áreas têm o mesmo parâmetro de comparação, além de que, esses últimos, possuem $23,75 \%$ na categoria de sobrepeso e $12,5 \%$ na categoria de obesidade (Gráfico 1).

Porém, comparando os docentes dos cursos da área da saúde e aqueles de cursos de outras áreas, de acordo com o gráfico 1 , com relação à obesidade e ao sobrepeso, os professores dos cursos da área da saúde possuem percentual de quase duas vezes a mais $(36,25 \%)$ que os dos cursos de outras áreas $(18,75 \%)$. Todavia, vale ressaltar que a quantidade de professores dos cursos da área da saúde é significativamente maior se comparado com aqueles dos cursos de outras áreas.

Gráfico 1 - Classificação do Índice de Massa Corporal (IMC) da amostra dos Professores Universitários do UNIPAM de acordo com os cursos que lecionam

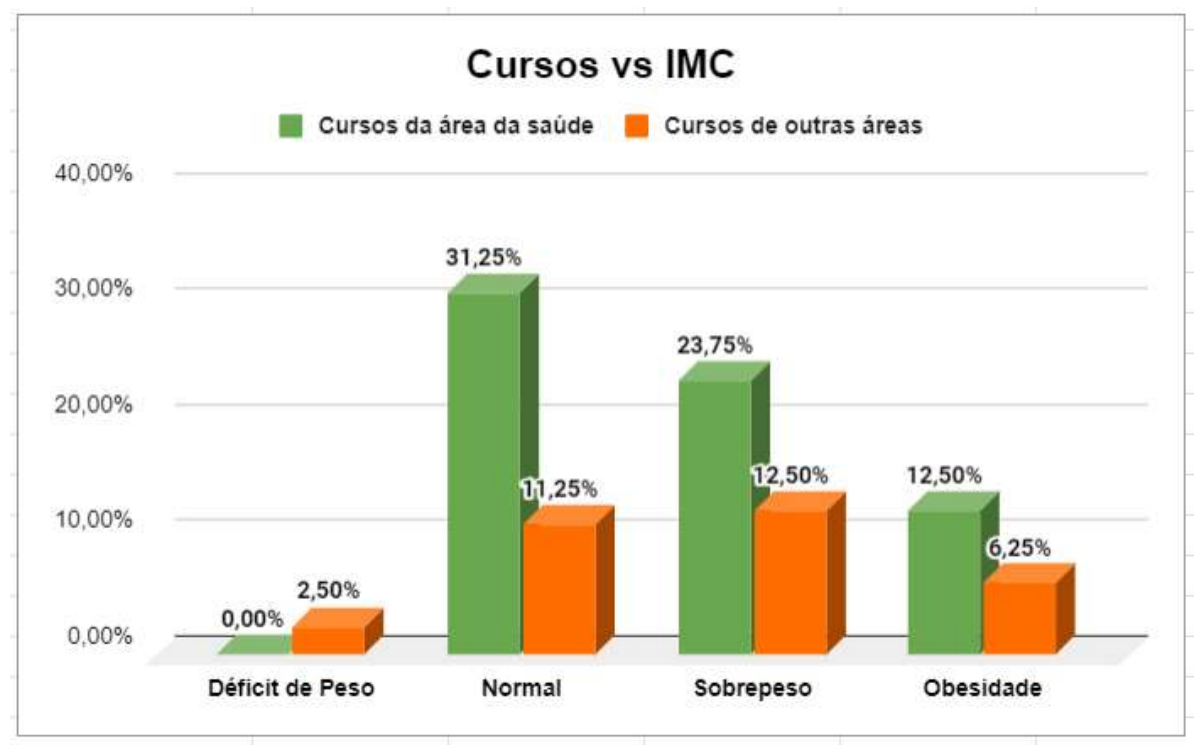

Fonte: Autores (2021).

Quanto a classificação do Gráfico 2, observa-se que, em relação à quantidade de horas que os professores do UNIPAM ficam sentados, de 4 a 7 horas é a que mais sobressaiu com 67,5\%. Considerando a quantidade de refeições por dia, 66,25\%, fazem de 3 a 4 refeições diárias e, no que diz respeito à prática de exercícios físicos, 75\% praticam exercícios físicos, sendo que, no nível de obesidade, os níveis de quem pratica e os de quem não pratica quase se equiparam, sendo $11,25 \%$ praticam e 7,5\% não praticam.

Em todos os gráficos, relacionados ao IMC, a classificação que obteve porcentagem maior foi a normal, resultando, portanto, em eutrofia. Esse resultado foi satisfatório, se comparado com outros estudos, como o de Cabral, et al. (2013), em que a prevalência geral de sobrepeso e obesidade entre os docentes foi de $33,8 \%$ e 13,8\%, respectivamente, sendo considerada uma porcentagem alta. 
Gráfico 2 - Classificação do Índice de Massa Corporal (IMC) da amostra dos Professores Universitários do UNIPAM de acordo com os cursos que lecionam
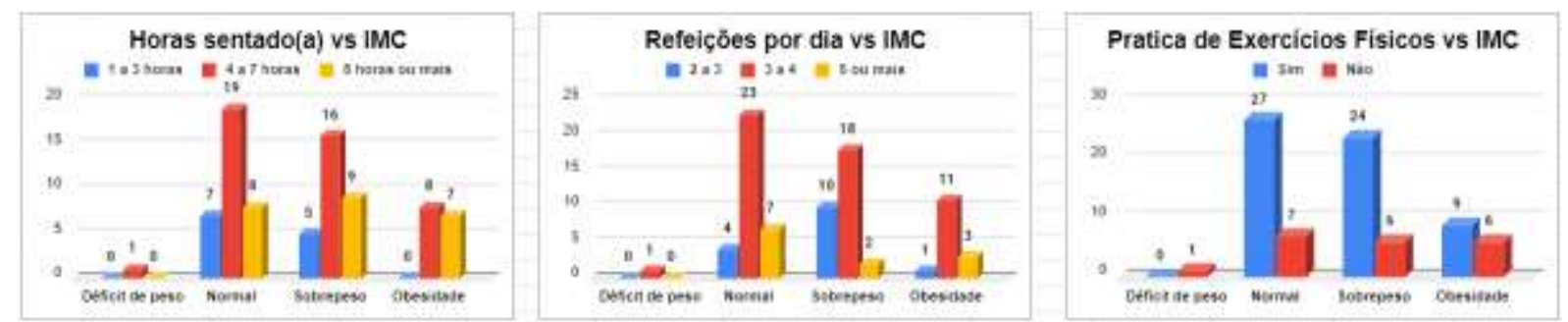

Fonte: Autores (2021).

A principal causa de obesidade é a alimentação de maneira inadequada ou excessiva. Uma pessoa com o peso ideal mantém o equilíbrio entre a quantidade de calorias ingeridas e a energia gasta ao longo do dia. Quando há excesso de alimentos e baixa atividade energética, ocorre o acúmulo de gordura. Por isso, o sedentarismo é o segundo fator importante que contribui para a obesidade (Brasil, 2020).

Diferentemente de Brasil (2020), o estudo apresentou, de acordo com as respostas dos professores universitários ao questionário, como primeiro fator para o desenvolvimento da obesidade, o sedentarismo (8,8\%), seguido da má alimentação $(6,25 \%)$ e do psicológico (1,25\%), sendo $16,3 \%$ os que se consideram obesos.

Além disso, o presente estudo constatou que apenas 42,5\% dos professores universitários não possuem nenhuma patologia, sendo que $57,5 \%$ possuem. Isso pode estar relacionado ao seu estilo de vida, à genética ou a fatores ambientais, mas independente da causa é um fator preocupante, já que uma patologia pode levar a outra.

De acordo com a Escala de Silhueta de Stunkard, o gráfico 3 compara as silhuetas com os sexos feminino e masculino, sendo que foi possível notar que a silhueta 4 prevaleceu no sexo feminino, com $20 \%$ e, no sexo masculino, as silhuetas 5 e 6 tiveram o mesmo percentual, sendo de $8,75 \%$, seguido pela silhueta 7 , com $5 \%$, o que demonstra maior predomínio do sexo masculino por silhuetas mais sinuosas, que pode caracterizar a obesidade.

Gráfico 3 - Classificação da Escala de Silhueta de Stunkard relacionada ao sexo.

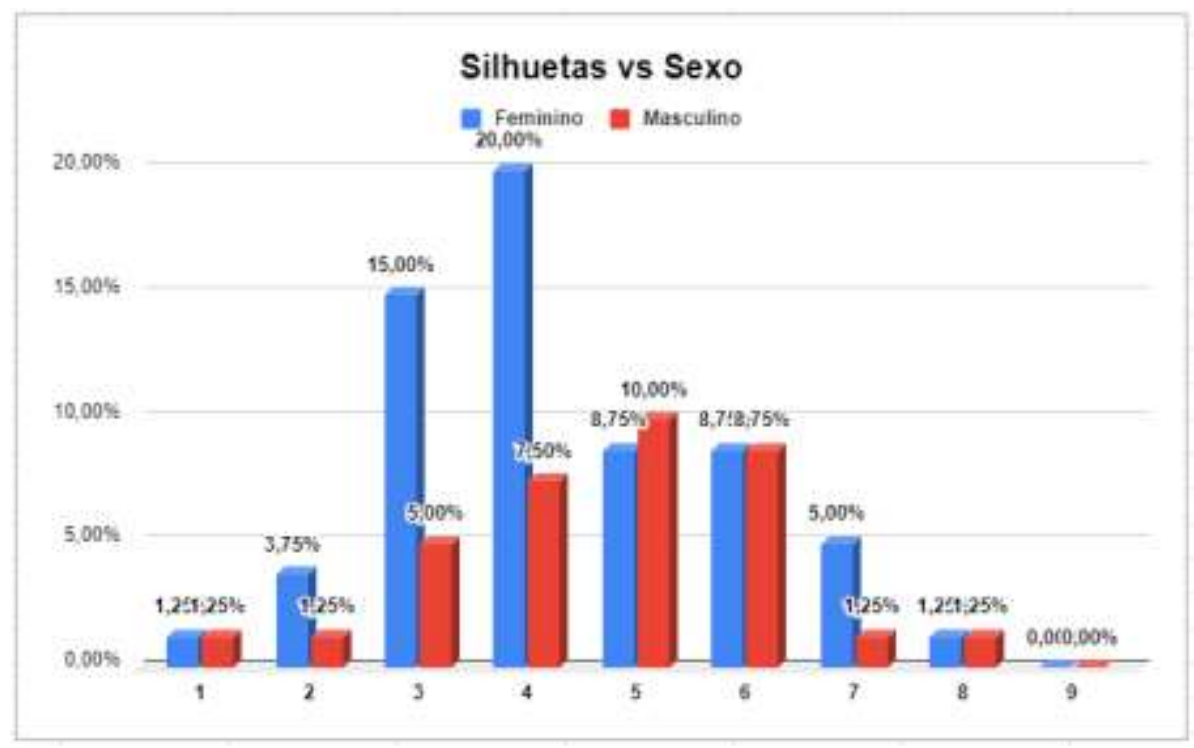

Fonte: Autores (2021). 
A mudança de comportamento ou de estilo de vida é uma das formas mais eficazes de tratamento para a obesidade. Dessas intervenções, a prática regular de exercícios físicos contribui para o controle do peso corporal e fornece vários benefícios, como melhora do nível de aptidão física e a prevenção ou o tratamento dos fatores de risco e das comorbidades (NHMRC, 2013).

Segundo a OMS (2020), para a redução de obesidade, as pessoas devem limitar o consumo de energia a partir de gorduras totais e açúcares, aumentar o consumo de frutas e de alimentos como verduras, legumes, grãos integrais e nozes, além de se envolver em atividade física regular (60 minutos por dia para crianças e 150 minutos por semana para adultos).

Inúmeros são os benefícios da realização de atividade física, como a melhora na autoestima, o aumento da força e do tônus muscular, além de estar associado na diminuição da depressão (Fermino, R. C., et al., 2010).

No presente estudo, $75 \%$ dos professores universitários, de ambos os sexos, praticam algum exercício físico e, dos que não estão praticando, 28,7\% já praticaram e 2,5\% nunca praticaram. Esse resultado é aceitável, já que mais da metade realizam algum exercício físico, porém é preocupante, visto que uma alta taxa não praticam, o que é extremamente prejudicial à saúde e pode acarretar várias patologias.

De acordo com a amostra total, o Gráfico 4 aponta que a maioria dos professores universitários consideram que a obesidade é considerada uma doença $(93,8 \%)$ e 6,3\% acham que a obesidade não é considerada uma doença. Além disso, de acordo com o questionamento se a obesidade é um dos fatores mais propensos para a morte perante a COVID-19, 82,5\% consideram que sim e 17,5\% consideram que não. Cientificamente, para as duas perguntas, é comprovado que ela é uma doença e é um dos fatores mais propensos para morte perante a nova patologia.

Gráfico 4 - Classificação da obesidade quanto doença e perante a COVID-19.

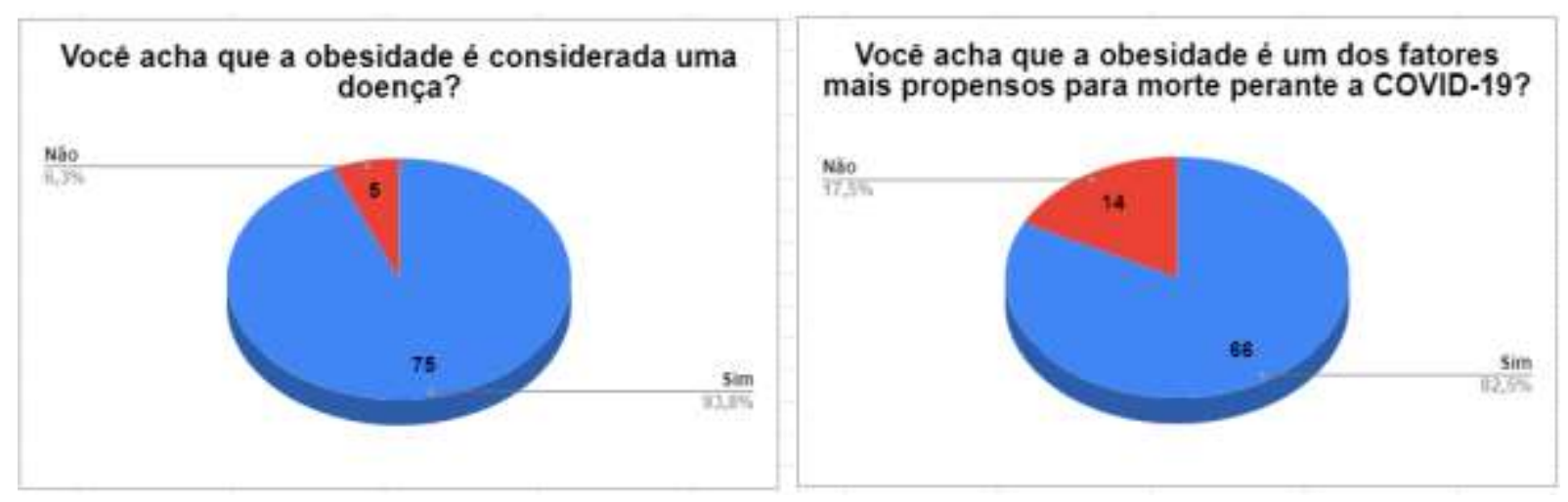

Fonte: Autores (2021).

Contrariando os 6,3\% que acham que a obesidade não é considerada uma doença, de acordo com o Ministério da Saúde (2011) a obesidade integra o grupo de doenças crônicas não transmissíveis (DCNT). As DCNTs são caracterizadas pelos seus múltiplos fatores de risco, longo período assintomático e, normalmente, lento, prolongado e permanente, com períodos de absolvição e de exacerbação, lesões celulares irreversíveis e evolução para diferentes graus de incapacidade ou para a morte.

Além disso, a infecção por SARS-CoV-2 torna-se especialmente grave aos pacientes que apresentam doenças consideradas comorbidades associadas à obesidade. O chamado "Choque de Pandemias" tem sido denominado para associar a problemática envolvida da infecção pelo novo coronavírus em pacientes acometidos por outra pandemia, mais antiga, crônica, multifatorial e insidiosa - a obesidade (Serfaty, 2020), observando-se, portanto, que as duas estão diretamente interligadas. Outro aspecto relevante é o quanto a relação entre a obesidade e suas comorbidades, abrange o estado inflamatório característico da sua fisiopatologia.

Dados internacionais mostram a alta frequência da obesidade entre os pacientes considerados graves internados em terapia intensiva por SARS-CoV-2, sendo que o risco de agravamento e de morte por COVID-19 é de até quatro vezes maior em 
pessoas com obesidade (Lighter, et al., 2020; Simonnet, et al., 2020). No Brasil, a obesidade é a principal comorbidade associada aos óbitos em pessoas com menos de 60 anos (Brasil, 2020).

O estudo realizado por Simonnet, et al., em 2020, demostrou que $85 \%$ dos pacientes com IMC $\geq 40 \mathrm{~kg} / \mathrm{m} 2$ que contraiu SARS-CoV-2, necessitaram de ventilação mecânica invasiva, enquanto que apenas $47 \%$ dos enfermos com IMC $<25 \mathrm{~kg} / \mathrm{m} 2$ apresentaram tal demanda. Assim, é notório que a gravidade da doença COVID-19 está diretamente relacionada ao aumento do IMC, e que a obesidade é um fator de risco para a morbimortalidade pela infecção por SARS-CoV-2, independente se estão ou não associadas a outras comorbidades.

O excesso de gordura corporal, principalmente da gordura visceral, ocasiona implicação da captação de glicose, resistência à insulina e diminuição da função das células beta pancreáticas, o que dificulta uma resposta metabólica pertinente, que é primordial numa condição de déficit imunológico, como ocorre nas infecções graves. Dessa maneira, o vírus SARS-CoV2 pode afetar diretamente a função das células beta pancreáticas, devido à sua interação com a enzima conversora de angiotensina 2 (ECA2). Ainda, pessoas com obesidade, além das comorbidades trombóticas e cardiovasculares, possuem diminuição da capacidade funcional e atenuação do sistema respiratório, da incursão diafragmática e hipoventilação, que, somado a um quadro de infecção pulmonar, acentua a condição de hipóxia pré-existente (Sattar, 2020).

A Figura 2 retrata os diferentes mecanismos que a obesidade pode estar contribuindo para o agravamento da infecção pelo SARs-COV2, sendo eles: desregulação do sistema imune, favorecimento da formação de trombo, aumento de inflamações, prejuízos do metabolismo da glicose, comprometimento do sistema respiratório e do sistema cardiovascular.

Figura 2 - Mecanismos fisiopatológicos que explicam o agravamento dos casos de COVID-19 em pacientes com obesidade.

\section{Como a obesidade contribui para a gravidade dos casos de COVID19?}

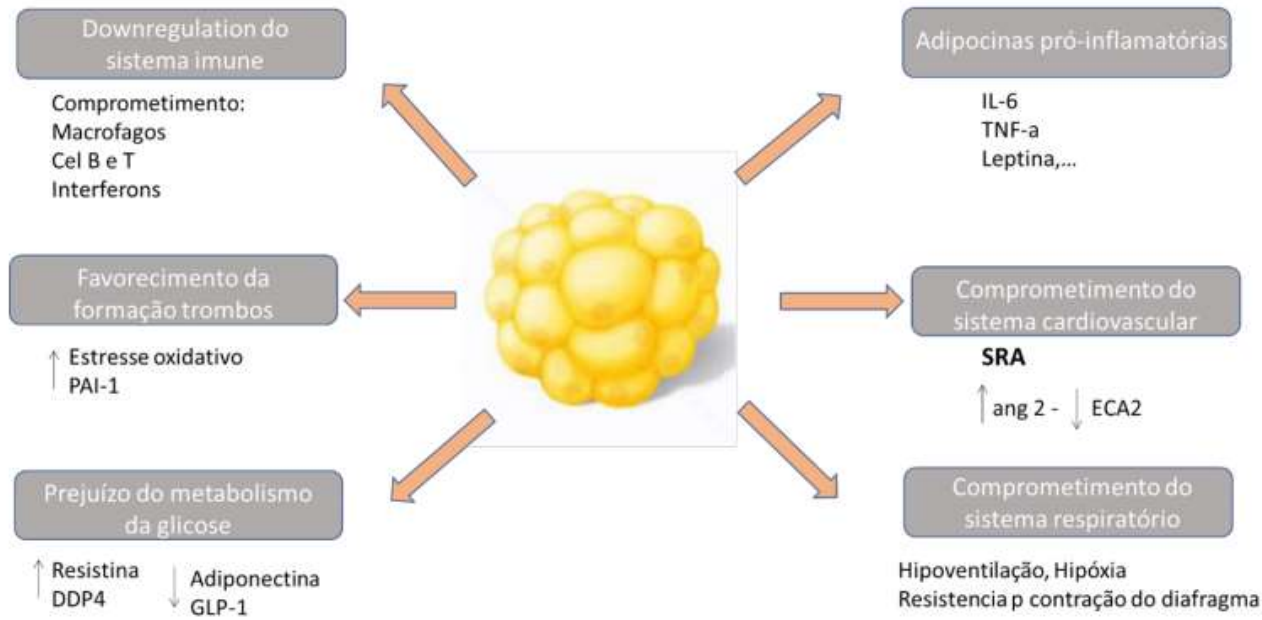

Nota: Ang2, angiotensina 2; DDP4, dipeptidil peptidase 4; ECA2, enzima conversora de angiotensina 2; GLP-1, peptideo semelhante a glucagon 1; IL-6, interleucina 6; PAI-1, inibidor do ativador de plasminogênio tipo 1; TNF-a, fator de necrose tumoral alfa. Fonte: Revista Gaúcha de Enfermagem (2021).

Ainda considerando a amostra total deste estudo, a maior parte dos professores universitários (86,3\%) julgam que os exercícios aeróbios auxiliam mais no combate à obesidade que os resistidos (Gráfico 5). 
Gráfico 5 - Classificação do tipo de exercício que mais auxilia no combate à obesidade.

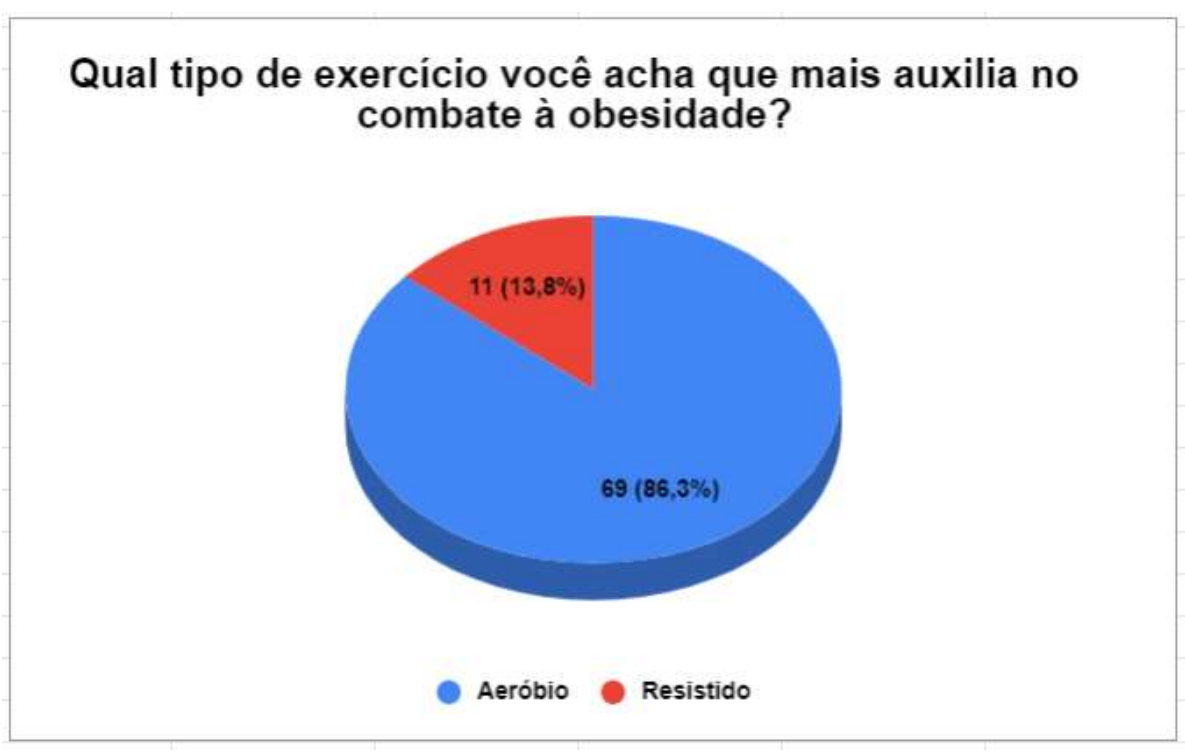

Fonte: Autores (2021).

Assim como no presente estudo, embora o treinamento resistido seja eficiente nos programas de atividade física, sua prática ainda é negligenciada, o que influencia à uma elevada recomendação por exercícios aeróbicos, inclusive para os praticantes obesos. Entretanto, um estudo indica que exercícios mais vigorosos têm maior eficácia por fornecer maior estabilidade, prevenção ou reabilitação de distúrbios musculoesqueléticos, ganho de massa muscular e perda de gordura corporal (Zemková, et al., 2017).

A prática do treinamento resistido, ou seja, exercício físico realizado com pesos adicionais, que podem ser objetos ou o próprio corpo, está relacionado na promoção da saúde e na prevenção de diversas comorbidades, como diabetes, hipertensão arterial e, principalmente, obesidade (Andrade, \& Fernandes, 2011; Arruda, et al., 2010; Callegari, et al., 2010).

Estudos têm demonstrado que os exercícios anaeróbicos são capazes de aumentar a massa corporal magra e, consequentemente, a taxa metabólica basal, além de promover um considerável gasto calórico durante o exercício físico e provocar um alto EPOC (consumo de oxigênio em excesso pós-exercício), o que proporciona que o organismo continue gastando calorias de maneira acelerada após o término dos exercícios, sendo que quanto maior a intensidade do exercício anaeróbico maior será o EPOC (Castinheiras \& Farinatti, 2010).

No estudo de Stegen, et al. (2011), por exemplo, 15 pacientes com obesidade mórbida foram avaliados, divididos em grupo experimental e grupo de controle, e submetidos a um programa de exercícios que incluiu aquecimento de dez minutos, 25 minutos de treinamento resistido (trabalho de flexão de cotovelo, extensão e flexão de joelhos, iniciando com duas séries de 15 repetições, com 60\% de repetição máxima e terminando com três séries de 15 repetições com 75\% de uma repetição máxima), 30 minutos de atividade aeróbica (iniciando com $60 \%$ e terminando o treinamento com $75 \%$ da frequência de reserva). Após quatro meses de intervenção os indivíduos foram reavaliados. Foi observada a manutenção da força muscular, o aumento da capacidade aeróbica e da capacidade funcional, além da redução da gordura corporal e da massa livre de gordura tanto no grupo experimental quanto no controle.

Em outro estudo realizado por Kirk, et al. (2009), que analisou o efeito de um programa de exercício resistido em 6 meses, com sessões de duração de aproximadamente 11 minutos e com exercícios de intensidade entre $85 \%$ e $90 \%$ de 1 repetição máxima (1RM), verificou que ocorreu um aumento na oxidação de gordura e na massa livre de gordura, bem como um aumento significativo no gasto energético, na massa corporal magra, na taxa metabólica de repouso, na taxa metabólica do sono. 
No treinamento muscular resistido, é recomendado que os exercícios envolvam grandes grupamentos musculares e sejam realizados de 2 a 3 vezes por semana, com carga entre 60\% a 70\% de 1RM em 3 séries de 12 a 15 repetições (Garvey, et al., 2016; Hansen, et al., 2018). Nessa perspectiva, o Colégio Americano de Medicina Esportiva (ACSM) recomenda que o treinamento muscular para iniciantes seja realizado de 1 a 3 séries, e para pessoas treinadas e atletas de 3 a 6 séries (ACSM, 2009). Em indivíduos não treinados, exercícios com alto volume (maior que três séries) não foram significativamente melhores que baixos volumes de treinamento (Teixeira, et al., 2017).

Além disso, o treinamento resistido deve ser composto por repetições dinâmicas de forma progressiva, com exercícios de ações musculares concêntricas, excêntricas e isométricas. Também devem ser incluídos exercícios unilaterais e bilaterais, de única e múltiplas articulações, sendo mais de um conjunto de exercício por grupo muscular. Recomenda-se que os exercícios sejam realizados com velocidade baixa ou moderada, em intervalos de 2 a 3 minutos para cada série (ACSM, 2009). A Figura 3 sintetiza os protocolos de exercícios físicos resistidos que podem ser aplicados em obesos.

Figura 3 - Protocolo de exercícios físicos resistidos para adultos e idosos com obesidade.
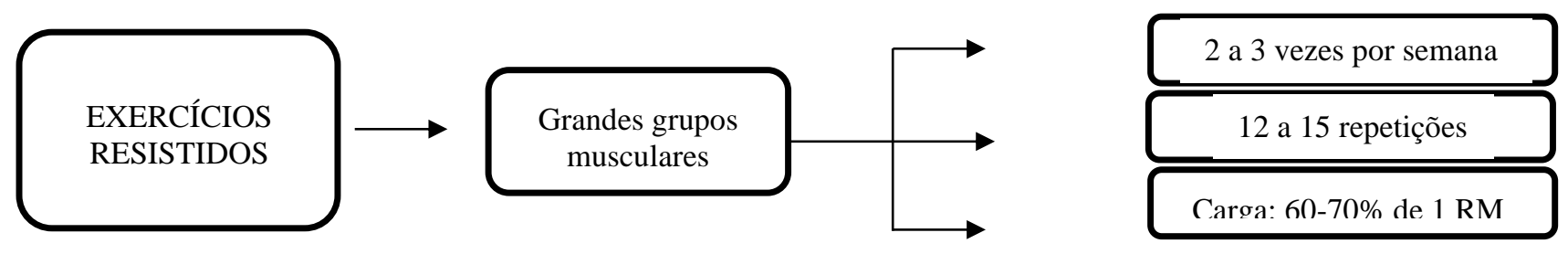

Fonte: Autores.

Portanto, o exercício resistido é um potente estímulo para aumentar a massa, força e potência muscular, podendo ajudar a preservar a musculatura, que tende a diminuir devido à dieta, maximizando a redução de gordura corporal (Faria, 2011). Além disso, o conhecido efeito de perda e ganho de peso em sequência tem um potencial efeito negativo e deve ser evitado por meio de programas de treinamento para manutenção do peso corporal em médio e longo prazo (Palm et al., 2017). Por esse motivo, as reavaliações periódicas ou anuais são importantes para monitoramento e supervisão da prevenção do ganho de peso (Garvey, et al., 2016; Hansen, et al., 2018).

Já o exercício aeróbico tem sido recomendado na literatura com alto grau de indicação, tanto para perda de peso quanto para melhora de aspectos relacionados ao quadro clínico dos pacientes obesos (Garvey, et al., 2016; Hansen, et al., 2018; Jensen, et al., 2013).

O exercício físico aeróbico de intensidade moderada, com aumento progressivo nos volumes de treinamento é o mais aconselhado, com a finalidade de aumentar o gasto calórico e de obter perda de peso clinicamente significativa (Garvey, et al., 2016; Hansen, et al., 2018; NHMRC, 2013). Entretanto, existem evidências que intervenções de alta intensidade produzem maiores perdas do que intervenções de intensidade moderada (Jensen, et al., 2013).

Quanto à prescrição recomendada, existem várias sugestões quanto à duração e à frequência dos exercícios físicos para obesos. As mais indicadas são a prescrição de exercício físico aeróbico superior a 150 minutos por semana divididos em 30 minutos em 5 dias da semana (Jensen, et al., 2013) e a prescrição progressiva do volume de exercício aeróbico superior a 250 minutos por semana até alcançar um nível de exercício físico elevado de forma permanente (Hansen, et al., 2018).

Apesar das discussões sobre a frequência de treinamento, sabe-se que as adaptações fisiológicas e metabólicas relacionadas a pratica de exercício físico são maiores quando realizadas diariamente, porém, é possível adaptar os exercícios quanto à intensidade, à frequência e à duração para que o paciente obtenha os benefícios do treinamento físico mesmo que essa prática não possa ser realizada todos os dias (Garvey, et al., 2016; Hansen, et al., 2018; Jensen, et al., 2013; NHMRC, 2013). 
Para que a redução do peso corporal evidencie benefícios clinicamente relevantes, são orientados programas de exercícios físicos por no mínimo seis meses, associados a um programa de restrição alimentar, com perdas maiores que 5-10\% do peso corporal. Realizar exercício aeróbico por no mínimo seis meses tem benefícios similares a 12 meses de treinamento (ABESO, 2016; Hansen, et al., 2018; Jensen, et al., 2013; NHMRC, 2013).

\section{Conclusão}

De acordo com os resultados obtidos no estudo constatou-se que a grande maioria dos professores universitários do UNIPAM se encontraram com o peso adequado, o que mostra que, mesmo sendo muito ocupados devido ao trabalho, praticam exercícios físicos e isso afeta positivamente em sua qualidade de vida, além de auxiliar na prevenção e no combate da obesidade e nos fatores de risco para morbidade.

Entretanto, mesmo com esse resultado satisfatório, tiveram alguns docentes que apresentaram sobrepeso e obesidade, sendo os dos cursos da área da saúde com maior percentual em relação ao IMC, que era estimado que eles, por serem da área da saúde, teriam uma vida mais saudável. Porém, o resultado pode ter sido interferido pela diferença de respostas, em que os da área da saúde foi expressivamente maior.

Portanto, mesmo que o presente estudo tenha sido adequado, vários outros não tiveram a mesma conclusão, apresentando a maioria dos professores universitários dos estudos com obesidade. Assim, mais estudos nessa área e com essa população são necessários, visto que essa patologia é um assunto preocupante, já que pode levar a outras morbidades e está crescendo de maneira exorbitante nos últimos anos.

\section{Referências}

ABESO. Associação Brasileira para o Estudo da Obesidade e da Síndrome Metabólica (2016). Diretrizes Brasileiras de Obesidade. (4a ed.).

ACSM. American College of Sports Medicine (2009). Progression models in resistance training for healthy adults. Medicine \& Science in Sports \& Exercise, $41(3), 687-708$.

Andrade, C. L. \& Fernandes, A. R. (2011). Treinamento com pesos e promoção da saúde em adultos: uma revisão sistemática da literatura nacional acerca dos métodos empregados em estudos científicos. Revista Colloquium Vitae, 3 (2), 59-66.

Arruda, D. P., et al. (2010). Relação entre treinamento de força e redução do peso corporal. Revista Brasileira de Prescrição e Fisiologia do Exercício, 4 (24).

Batsis, J. A., et al. (2017). Weight loss interventions in older adults with obesity: a systematic review of randomized controlled trials since 2005 . Journal of the American Geriatrics Society, 65 (2), 257-268.

Brasil. Governo do Brasil (2020). Habilitar estabelecimento de saúde como serviço de assistência de alta complexidade ao indivíduo com obesidade.

Brasil. Ministério da Saúde, Secretaria de Vigilância em Saúde (2020). Boletim Epidemiológico 09 - COE Coronavírus.

Brasil. Ministério da Saúde, Secretaria de Vigilância em Saúde (2011). Plano de ações estratégicas para o enfrentamento das doenças crônicas não transmissíveis (DCNT) no Brasil 2011-2022. Brasília: Ministério da Saúde.

Cabral, L. L., et al. (2013). Estágios de mudança de comportamento para a prática de atividades físicas e indicadores de obesidade em professores universitários. Revista do Departamento de Educação Física e Saúde e do Mestrado em Promoção da Saúde da Universidade de Santa Cruz do Sul / Unisc, 14 (4), 181-185.

Callegari, G. A., et al. (2010). Perfil antropométrico dos indivíduos iniciantes na prática da musculação. Revista Brasileira de Prescrição e Fisiologia do Exercício, 4 (24), 618-624.

Castinheiras, A. G. N. \& Farinatti, P. T. V. (2010). Efeito da Manipulação das variáveis do treinamento de força sobre o consumo de oxigênio pós-exercício (EPOC). Revisão Crítica. Revista Brasileira de Nutrição Clínica, 25 (1), 48-56.

Cavalcanti, C. B. S., et al. (2010). Obesidade Abdominal em Adolescentes: Prevalência e Associação com Atividade Física e Hábitos Alimentares. Arquivos Brasileiros de Cardiologia, Rio de Janeiro, 94 (3), 371-377.

Digital, Olivas (2021). Mapa da obesidade. Associação Brasileira para o Estudo da Obesidade e da Síndrome Metabólica- ABESO.

Digital, Olivas (2021). Obesidade e sobrepeso. Associação Brasileira para o Estudo da Obesidade e da Síndrome Metabólica- ABESO.

Estrela, C. (2018). Metodologia Científica: Ciência, Ensino, Pesquisa. Editora Artes Médicas. 
Faria, T. C. S. (2011). Contribuição da musculação para o processo de emagrecimento em mulheres. Trabalho de Conclusão de Curso - Escola de Educação Física, Fisioterapia e Terapia Ocupacional da Universidade Federal de Minas Gerais (UFMG), Belo Horizonte.

Fermino, R. C., et al. (2010). Motivos para prática de atividade física e imagem corporal em frequentadores de academia. Revista Brasileira de Medicina do Esporte, 16 (1).

Fernandez, A. C., et al. (2004). Influence of aerobic and anaerobic training on the body fat mass in obese adolescents. Revista Brasileira de Medicina do Esporte, $10(1), 159-164$.

Garvey, W. T., et al. (2016). American Association of Clinical Endocrinologists and American College of Endocrinology comprehensive clinical practice guidelines for medical care of patients with obesity. Endocrine Practice, 22 (3), 1-203.

Hansen, D., et al. (2018). Exercise prescription in patients with different combinations of cardiovascular disease risk factors: a consensus statement from the EXPERT working group. Sports Medicine, 48 (8), 1781-1797.

Jensen, M. D., et al. (2013). AHA/ACC/TOS Guideline for the management of overweight and obesity in adults: overweight and obesity in adults: a report of the American College of Cardiology/ American Heart Association task force on practice guidelines and The Obesity Society. Circulation, 129 (252), $102-138$.

Kirk, E. P., et al. (2009). Minimal resistance training improves daily energy expenditure and fat oxidation. Medicine \& Science Sports \& Exercise, 41 (5), $1122-$ 1129 .

Lighter, J., et al. (2020). Obesity in patients younger than 60 years is a risk factor for Covid-19 hospital admission. Clinical Infectious Diseases, 71 (15).

Manna, P. \& Jain, S. K. (2015). Obesity, oxidative stress, adipose tissue dysfunction, and the associated health risks: causes and therapeutic strategies. Metab Syndr Relat Disord, 12 (10), 423-444.

NHMRC. National Health and Medical Research Council (2013). Clinical practice guidelines for the management of overweight and obesity in adults, adolescents and children in Australia. Melbourne: National Health and Medical Research Council.

Palm, I., et al. (2017). Body weight cycling with identical diet composition does not affect energy balance and has no adverse effect on metabolic health parameters. Nutrients, $9(10), 1149-1160$.

PNS. Pesquisa Nacional de Saúde 2019. Instituto Brasileiro de Geografia e Estatística (IBGE) (2020). Atenção primária à saúde e informações antropométricas. Diretoria de Pesquisas. Coordenação de Trabalho e Rendimento-COREN. 2.

PNS. Pesquisa Nacional de Saúde 2019. Instituto Brasileiro de Geografia e Estatística (IBGE) (2020). Um em cada quatro adultos do país estava obeso em 2019; Atenção Primária foi bem avaliada. Estatísticas Sociais.

Poehlman, E. T., et al. (2000). Effects of resistance training and endurance training on insulin sensitivity in nonobese, young women: a controlled randomized trial. The Journal of Clinical Endocrinology \& Metabolism, 85 (7), 2463-2468.

Sattar, N. \& Mcinnes, I. B.; Mcmurray, J. J.V. (2020). Obesity a risk factor for severe COVID-19 infection: multiple potential mechanisms. Circulation, v. 142 $(1), 4-6$.

Serfaty, F. M. (2020). Covid-19 e obesidade: duas pandemias. Medscape.

Simonnet, A., et al. (2020). High prevalence of obesity in severe acute respiratory syndrome coronavirus-2 (SARS-CoV-2) requiring invasive mechanical ventilation. Obesity (Silver Spring), 28 (7).

Stegen, S., et al. (2011). Physical Fitness in Morbidly Obese Patients: Effect of Gastric Bypass Surgery and Training. Obes Surg, 21 (1), 61-70.

Teixeira, C. V. L. S., et al. (2017). Effect of resistance training set volume on upper body muscle hypertrophy: are more sets really better than less? Clinical Physiology and Functional Imaging, 38 (5), 727-732.

WHO. World Health Organization (2020). Obesity and overweight.

Zabatiero, J., et al. (2015). Beliefs, barriers and facilitators to physical activity in bariatric surgery candidates. Obesity Surgery, 26 (5), $1097-1109$.

Zemková, E., et al. (2017). Muscular power during a lifting task increases after three months of resistance training in overweight and obese individuals. Sports, $5(2), 35$. 